\title{
RBF Neural Network Adaptive Control Strategy based on Sub- Block Approximation Algorithm
}

\author{
Yongfeng Cui ${ }^{1, *}$ and Chong Tian ${ }^{2}$ \\ ${ }^{1}$ School of Computer Science and Technology, Zhoukou Normal University, \\ Zhoukou 466001, China \\ ${ }^{2}$ School of Mathematics and Statistics, Zhoukou Normal University, Zhoukou \\ Henan 466001, China \\ cuiyf@zknu.edu.cn
}

\begin{abstract}
Aiming at the uncertainties that existed in the dynamic model of robot may cause instability.This paper has proposed a RBF neural network adaptive control strategy based on sub-block approximation algorithm, in this strategy, sliding model control was used to control trajectory of the joints of robot, and utilize the RBF neural network to approximate the each uncertain in the dynamic model of robot. In order to verify the validity of the control algorithm, we realized the RBF neural network adaptive control strategy based on sub-block approximation algorithm in MATLAB. The simulation results show that compared with the RBF neural network adaptive control strategy based on integral approximation for uncertainness, the proposed control method has features with good position tracking, the smooth movement, effective approaches, etc.
\end{abstract}

Keywords: Sliding model control, Model approximation, RBF neural network, Subblock approximation, Numerical simulation

\section{Introduction}

In recent years, there are some researching results about the trajectory tracking of robot[1].Seeing From the control system of manipulator, there is coupling between each joint of manipulator, the existed coupling may make the robot more strong coupling, highly nonlinear, time-varying and so forth[2].

So, an effective intelligent control strategy for robot has always been a hotspot in research of robot control[3]. The traditional model-based control algorithm for robot is to be implemented based on the accurate mathematical model, but there have existed strong coupling, highly nonlinear, time-varying and so forth in the robot system. The model parameters change along with its position, posture and load changes, at the same time there has interference, model uncertainties and other factors in the system, due to the mentioned uncertainties, it is difficult to convergence trajectory tracking error[4], in that case, it made the design of the controller into a difficult task .

In order to achieve the online compensation control for the uncertainties, control algorithms have been proposed to achieve the online compensation control for the uncertainties. Such as, adaptive control, SMC, improved-PID and so forth. Adaptive control focuses on containing parameter uncertainties that can be linearized, but it has strict real-time, complex implementation, the adaptive control is difficult to guarantee the stability of system and achieve the control performance especially in the condition that there existed parameter uncertainties [3-5].

Robust control has better control effect, this method needs to know that the upper bound of the uncertainty, but it is hard to get its upper bounds of the uncertainties of the manipulator in the actual applications [6-7].Considering the defect of above methods, so 
as to Ensures the control system has good dynamic performance and robust performance, many scholars have made the intelligent control method, robust control and adaptive control together to control the system [8-10].

Artificial intelligence neural network control has the good ability to approximate the highly nonlinear, it has proposed a new way to solve the problem of the robot control. In that case, intelligent control based on neural network has been widely used in the precision control of robot system with uncertainties [11-14].

In this presentation, aiming at the uncertainness that existed in the dynamic model of robot may cause instability. This paper has proposed a RBF neural network adaptive control strategy based on sub-block approximation algorithm, in this strategy, sliding model control was used to control trajectory of the joints of robot, and utilize the RBF neural network to approximate the each uncertain in the dynamic model of robot. In order to verify the validity of the control algorithm, we realized the RBF neural network adaptive control strategy based on sub-block approximation algorithm in MATLAB. The simulation results show that compared with the RBF neural network adaptive control strategy based on integral approximation for uncertainness, the proposed control method has features with good position tracking, the smooth movement, effective approaches, etc.

\section{Problem Statement}

Supposed that the equation for manipulator with $\mathrm{n}$ joints is:

$$
M(q) \ddot{q}+C(q, \dot{q}) \dot{q}+G(q)+F(\dot{q})+\tau_{d}=\tau
$$

Where, $M(q)$ is positive definite inertia matrix with $n \times n, C(q, \dot{q})$ is inertia matrix with $n \times n, G(q)$ is inertia vector with $n \times 1, F(\dot{q})$ is the friction force, $\tau_{d}$ is unknown external disturbance, $\tau$ is input of control.

Define the position tracking error as :

$$
e(t)=q_{d}(t)-q(t)
$$

Define error function as :

$$
r=\dot{e}+\Lambda e
$$

Where $\Lambda=\Lambda^{\mathrm{T}}>0$, then

$$
\begin{aligned}
\dot{\mathrm{q}}= & -r+\dot{\mathrm{q}}_{d}+\Lambda e \\
M \dot{\mathrm{r}}= & M\left(\ddot{\mathrm{q}}_{d}-\ddot{\mathrm{q}}+\Lambda e\right)=M\left(\ddot{\mathrm{q}_{d}}+\Lambda e\right)-M \ddot{\mathrm{q}} \\
& =M\left(\ddot{\mathrm{q}_{d}}+\Lambda e\right)+C \dot{\mathrm{q}}+G+F+\tau_{d}-\tau \\
& =M\left(\ddot{\mathrm{q}}_{d}+\Lambda \dot{e}\right)-C r+C\left(\dot{\mathrm{q}}_{d}+\Lambda \dot{e}\right)+G+F+\tau_{d}-\tau \\
& =-C r-\tau+f+\tau_{d}
\end{aligned}
$$

Where: $f(x)=M\left(\ddot{\mathrm{q}}_{d}+\Lambda \dot{e}\right)-C r+C\left(\dot{\mathrm{q}}_{d}+\Lambda \dot{e}\right)+G+F$.

In practice, the model uncertainties $f$ is unknown, so we need to approximate the uncertainties. 
In this paper we adopt the RBF to approximate the uncertainties, according to equation of $f(x)$, the input of $\mathrm{RBF}$ network is:

$$
x=\left[\begin{array}{ccccc}
e^{T} & e^{T} & q_{d}^{T} & \dot{q}_{d}^{T} & \ddot{q}_{d}^{T}
\end{array}\right]
$$

Define the control law as:

$$
\tau=\hat{f}+K_{v} r
$$

Where, $f(x)$ is estimation value for $f(x)$.

The control law type (6) generation type (4), put the (6) into equation (4), we can get that:

$$
M \dot{\mathrm{r}}=-C r-\hat{f}-K_{v} r+f+\tau_{d}=-\left(K_{v}+C\right) r+\hat{f}+\tau_{d}=-\left(K_{v}+C\right) r+\zeta_{0}
$$

Where $f=f-\hat{f}, \zeta_{0}=f+\tau_{d}$

Define Lyapunov function as:

$$
L=\frac{1}{2} r^{T} M r+\frac{1}{2} f^{2}
$$

Then

$$
\begin{gathered}
\dot{L}=r^{T} M \dot{M}+\frac{1}{2} r^{T} \dot{M} r+f f=-r^{T} K_{v} r+\frac{1}{2} r^{T}(\dot{M}-2 C) r+r^{T} \zeta_{0}+f f \\
\dot{L}=r^{T} \zeta_{0}-r^{T} K_{v} r+f f
\end{gathered}
$$

When the $K_{v}$ is constant, the stability of control system rely on the $\zeta_{0}$ and $f$. The ideal algorithm for RBF network by using RBF network to approximate uncertainties is:

$$
\begin{gathered}
\phi_{i}=g\left(\left\|x-c_{i}\right\|^{2} / \sigma_{i}^{2}\right), i=1,2, \cdots, n \\
f(x)=W \varphi(x)+\varepsilon
\end{gathered}
$$

Where $x$ is input of RBF, $\varphi=\left[\begin{array}{llll}\phi_{1} & \phi_{2} & \cdots & \phi_{1}\end{array}\right], \varepsilon$ is approximation error by using RBF neural network.

\section{Controller based on RBF with Overall Approach}

\subsection{The Design of the Controller}

Adopt the RBF neural network to approach the $f$, then the output of the RBF neural network is :

$$
\hat{f}(x)=\hat{W}^{T} \varphi(x)
$$

Taken

$$
\stackrel{\square}{\mathrm{W}}=\mathrm{W}-\hat{\mathrm{W}},\|W\|_{F} \leq W_{\max }
$$

Define the control law as:

$$
\tau=\hat{f}(x)+K_{v} r-v=\hat{W^{T}} \varphi(x)+K_{v} r-v
$$

Where $v$ is the robust item that is used to overcome the approximation error by using RBF neural network 
Put the control law (12) into equation (4), then:

$$
M \dot{\mathrm{r}}=-\left(K_{v}+C\right) r+w^{T} \varphi(x)+\left(\varepsilon+\tau_{d}\right)+v=-\left(K_{v}+C\right) r+\zeta_{1}
$$

Where $\zeta_{1}=w^{T} \varphi(x)+\left(\varepsilon+\tau_{d}\right)+v$.

\subsection{Stability and Convergence Analysis}

If there have $v(t), \varepsilon$ and $\tau_{d}$ will cause different convergence.

(1)Take $v(t)=0$, there have $\varepsilon$ and $\tau_{d}$

Define Lyapunov function as:

$$
L=\frac{1}{2} r^{T} M r+\frac{1}{2} \operatorname{tr}\left(W^{T} F^{-1} W\right)
$$

Then

$$
\dot{L}=r^{T} M \dot{r}+\frac{1}{2} r^{T} \dot{M} r+\operatorname{tr}\left(\square^{T} F^{-1} \dot{\dot{\square}}\right)
$$

Put (14) into (16), we can obtain:

$$
\dot{L}=-r^{T} K_{v} r+\frac{1}{2} r^{T}(\dot{M}-2 C) r+\operatorname{tr} W^{T}\left(F^{-1} \dot{\dot{\square}}+\varphi r^{T}\right)+r^{T}\left(\varepsilon+\tau_{d}\right)
$$

Considering the robot features, we take:

$$
W=-F \varphi r^{T}
$$

Then, the neural network adaptive law is:

$$
\begin{gathered}
\dot{\hat{W}}=F \varphi r^{T} \\
\dot{L}=-r^{T} K_{v} r+r^{T}\left(\varepsilon+\tau_{d}\right) \leq-\mathrm{K}_{v \min }\|r\|^{2}+\left(\varepsilon_{N}+b_{d}\right)\|r\|
\end{gathered}
$$

where $\|\varepsilon\| \leq \varepsilon_{N},\left\|\tau_{d}\right\| \leq b_{d}$.

When meet the convergence condition of the following $L \leq 0$ :

$$
\|r\|>\left(\varepsilon_{N}+b_{d}\right) / \mathrm{K}_{v \text { min }}
$$

(2)Take $v(t)=0, \varepsilon=0, \tau_{d}=0$

Define Lyapunov function as:

$$
L=\frac{1}{2} r^{T} M r+\frac{1}{2} \operatorname{tr}\left(\stackrel{\square}{W}^{T} F^{-1} W\right)
$$

Then the neural network adaptive and control law are:

$$
\begin{gathered}
\tau=\stackrel{\square}{ }^{T} \varphi(x)+K_{v} r \\
\dot{\hat{W}}=F \varphi r^{T}
\end{gathered}
$$

By equation (3), we obtain: 


$$
\begin{gathered}
M \dot{\mathrm{r}}=-\left(K_{v}+C\right) r+w^{T} \varphi(x) \\
\dot{\mathrm{L}}=r^{T} M \dot{\mathrm{r}}+\frac{1}{2} \dot{M} r=-r^{T} K_{v} r
\end{gathered}
$$

Due to the $\ddot{\mathrm{L}}=-r^{T} K_{v} \dot{r}$, then $\ddot{\mathrm{L}}_{\mathrm{i}}$ bounded, according to the barbalat lemma $\dot{\mathrm{L}} \rightarrow 0$, so, $\mathrm{r} \rightarrow 0$.

(3)Take $v(t)=0$, and there have $\varepsilon$ and $\tau_{d}$, also the adaptive law is

Define Lyapunov function and control law same as (22) and (23), the adaptive law is:

$$
\hat{\hat{W}}=F \varphi r^{T}-k F\|r\| \hat{W}
$$

According to (17), there has :

$$
\dot{L}=-r^{T} K_{v} r+\frac{1}{2} r^{T}(\dot{M}-2 C) r+\operatorname{tr} W^{T}\left(F^{-1} W+\varphi r^{T}\right)+r^{T}\left(\varepsilon+\tau_{d}\right)
$$

Put (27) into (28), then

Due to

$$
\begin{aligned}
\dot{L} & =-r^{T} K_{v} r+\operatorname{tr} \stackrel{\square}{W}^{T}\left(-\varphi r^{T}+k\|r\| \bar{w}+\varphi r^{T}\right)+r^{T}\left(\varepsilon+\tau_{d}\right) \\
& =-r^{T} K_{v} r+k\|r\| \operatorname{tr} W^{T}(w-\bar{w})+r^{T}\left(\varepsilon+\tau_{d}\right)
\end{aligned}
$$

Because

$$
\begin{gathered}
\operatorname{tr} \stackrel{\square}{ }^{T}(W-\bar{W})=(W, W)_{F}-\|W\|_{F}^{2} \leq\|w\|_{F}\|W\|_{F}-\|W\|_{F}^{2} \\
\dot{L} \leq-K_{v \min }\|r\|^{2}+k\|r\|\|w\|_{F}\left(W_{\max }-\|W\|_{F}\right)+\left(\varepsilon+\tau_{d}\right)\|r\| \\
=-\|r\|\left(K_{v \text { min }}\|r\|+k\|w\|_{F}\left(\|W\|_{F}-W_{\max }\right)-\left(\varepsilon+\tau_{d}\right)\right)
\end{gathered}
$$

$$
\begin{aligned}
& \left(K_{v \min }\|r\|+k\|w\|_{F}\left(\|W\|_{F}-W_{\max }\right)-\left(\varepsilon+\tau_{d}\right)\right) \\
& =\mathrm{k}\left(\|W\|_{F}-W_{\max } / 2\right)^{2}-k W_{\max }^{2} / 4+K_{v \min }\|r\|-\left(\varepsilon+\tau_{d}\right)
\end{aligned}
$$

So in order to make $L<0$, we need that:

$$
\|r\|>\frac{k W_{\max }^{2}+\left(\varepsilon+\tau_{d}\right)}{K_{v \min }}
$$

or

$$
\|W\|_{F}>W_{\max } / 2+\sqrt{k W_{\max }^{2} / 4+\left(\varepsilon+\tau_{d}\right) / k}
$$

(4)There have $\varepsilon$ and $\tau_{d}$, and also consider the robust item $v(t)$

We design the robust item $v(t)$ as: 


$$
v=-\left(\varepsilon+\tau_{d}\right) \operatorname{sgn}(r)
$$

Take the (13) as control law, and adaptive law for RBF is(19).

Define Lyapunov function as:

$$
L=\frac{1}{2} r^{T} M r+\frac{1}{2} \operatorname{tr}\left(\stackrel{\square}{W}^{T} F^{-1} W\right)
$$

Then

$$
\dot{L}=r^{T} M \dot{r}+\frac{1}{2} r^{T} \dot{M} r+\operatorname{tr}\left(W^{T} F^{-1} \dot{\dot{\square}}\right)
$$

because

$$
r^{T}\left(\varepsilon+\tau_{d}+v\right)=r^{T}\left(\varepsilon+\tau_{d}\right)+r^{T} v=r^{T}\left(\varepsilon+\tau_{d}\right)-\|r\|\left(\varepsilon+\tau_{d}\right) \leq 0
$$

Then

$$
\dot{L} \leq 0
$$

\section{RBF Neural Network Adaptive Control Strategy Based On Sub- Block Approximation Algorithm}

\subsection{Control Law}

Take the control law as:

$$
\tau=\hat{W}^{T} \varphi(x)+K_{v} r-v
$$

Take equation (35) as robust item $v$.

By equation (4), $f(x)$ can be written as:

$$
f(x)=M(q) \zeta_{1}(t)+C(q, \dot{q}) \zeta_{2}(t)+G(q)+F(\dot{q})
$$

Where $\zeta_{1}(t)=\ddot{q}_{d}+\Lambda \dot{e}, \zeta_{2}(t)=\dot{q}_{d}+\Lambda \mathrm{e}$.

Adopt the RBF neural network to approach the uncertainties based on sub-block approximation algorithm, we can approximate the each factors based on sub-block approximation algorithm:

$$
\begin{aligned}
& \hat{M}(q)=\hat{M}_{M}^{T} \varphi_{M}(q), \quad \hat{C}(q, \dot{q})=\hat{M}_{V}^{T} \varphi_{V}(q, \dot{q}), \\
& \hat{G}(q)=\hat{M}_{G}^{T} \varphi_{G}(q), \quad \hat{F}(\dot{q})=\hat{M}_{F}^{T} \varphi_{F}(\dot{q}) .
\end{aligned}
$$

Then

$$
\hat{f}(x)=\left[\begin{array}{llll}
\hat{W}_{M}^{T} \zeta_{1}(t) & \hat{W}_{V}^{T} \zeta_{2}(t) & \hat{W}_{G}^{T} & \hat{W}_{F}^{T}
\end{array}\right]\left[\begin{array}{c}
\varphi_{M} \\
\varphi_{V} \\
\varphi_{G} \\
\varphi_{F}
\end{array}\right]
$$


Where $\varphi(x)=\left[\begin{array}{c}\varphi_{M} \\ \varphi_{V} \\ \varphi_{G} \\ \varphi_{F}\end{array}\right], \hat{W}^{T}=\left[\begin{array}{cccc}\hat{W}_{M} & \hat{W}_{V}^{T} & \hat{W}_{G}^{T} & \hat{W}_{F}^{T} \\ & & & \end{array}\right]$ 。

Define the adaptive law as:

$$
\begin{aligned}
& \hat{W}_{M}=F_{M} \varphi_{M} r^{T}-k_{M} F_{M}\|r\| \hat{W}_{M} \\
& \dot{\hat{W}}_{V}=F_{V} \varphi_{V} r^{T}-k_{V} F_{V}\|r\| \hat{W}_{V} \\
& \hat{\hat{W}}_{G}=F_{G} \varphi_{G} r^{T}-k_{G} F_{G}\|r\| \hat{W}_{G} \\
& \hat{\hat{W}}_{F}=F_{F} \varphi_{F} r^{T}-k_{F} F_{F}\|r\| \hat{W}_{F}
\end{aligned}
$$

Where $k_{M}>0, k_{V}>0, k_{G}>0, k_{F}>0$.

\subsection{Stability Analysis}

Define Lyapunov function as:

$$
\begin{aligned}
L= & \frac{1}{2} r^{T} M r+\frac{1}{2} \operatorname{tr}\left(W_{M}^{T} F_{M}^{-1} W_{M}\right)+\frac{1}{2} \operatorname{tr}\left(W_{V}^{T} F_{V}^{-1} W_{V}\right) \\
+ & \frac{1}{2} \operatorname{tr}\left(\mathbb{W}_{G}^{T} F_{G}^{-1} W_{G}\right)+\frac{1}{2} \operatorname{tr}\left(W_{F}^{T} F_{F}^{-1} W_{F}\right) \\
\dot{L}=r^{T} M & \dot{r}+\frac{1}{2} r^{T} \dot{M} r+\operatorname{tr}\left(W_{M}^{T} F_{M}^{-1} W_{M}\right)+\operatorname{tr}\left(W_{V}^{T} F_{V}^{-1} W_{V}\right) \\
& +\operatorname{tr}\left(W_{G}^{T} F_{G}^{-1} W_{G}\right)+\operatorname{tr}\left(W_{F}^{T} F_{F}^{-1} W_{F}\right)
\end{aligned}
$$

Put (14)into (49), then

$$
\begin{aligned}
& \dot{L}=-r^{T} K_{v} r+\frac{1}{2} r^{T}(\dot{M}-2 C) r+r^{T}\left(\varepsilon+\tau_{d}\right)+r^{T} v+\operatorname{tr} \mathbb{W}_{M}^{T}\left(F_{M}^{-1} W_{M}+\varphi_{M} r^{T}\right) \\
& +\operatorname{tr} W_{V}^{T}\left(F_{V}^{-1} W_{V}+\varphi_{V} r^{T}\right)+\operatorname{tr} W_{G}^{T}\left(F_{G}^{-1} W_{G}+\varphi_{G} r^{T}\right)+\operatorname{tr} W_{F}^{T}\left(F_{F}^{-1} W_{F}+\varphi_{F} r^{T}\right)
\end{aligned}
$$

Considering the robot features, and put the adaptive law in to the (44)-(47),then:

$$
\begin{aligned}
& \dot{L}=-r^{T} K_{v} r+k_{M}\|r\| \operatorname{tr} \stackrel{\square}{M}_{M}^{T}\left(W_{M}-W_{M}\right)+k_{V}\|r\| \operatorname{tr} W_{V}^{T}\left(W_{V}-W_{V}\right) \\
& +k_{G}\|r\| \operatorname{tr} \stackrel{\square}{W}_{G}^{T}\left(W_{G}-W_{G}\right)+k_{F}\|r\| \operatorname{tr} W_{F}^{T}\left(W_{F}-W_{F}\right)+r^{T}\left(\varepsilon+\tau_{d}\right)+r^{T} v
\end{aligned}
$$

Due to 


$$
\operatorname{tr}^{\square}(W-W)=(W, W)-\|W\|_{F}^{2} \leq\|W\|_{F}\|W\|_{F}-\|W\|_{F}^{2}
$$

Considering (34), then

$$
\begin{aligned}
\dot{L} \leq & -K_{v \min }\|r\|^{2}+k_{M}\|r\|\left\|_{M}\right\|\left(W_{F}-\left\|W_{M}\right\|_{F}\right)+k_{V}\|r\|\left\|_{F} W_{V}\right\|_{F}\left(W_{V \max }-\left\|W_{V}\right\|_{F}\right) \\
& +k_{G}\|r\|\left\|_{G}\right\|\left(W_{F}\left(W_{G \max }-\left\|W_{G}\right\|_{F}\right)+k_{F}\|r\|\left\|_{F}\right\| \|_{F}\left(W_{F \max }-\left\|W_{F}\right\|_{F}\right)\right. \\
= & -\|r\|\left[\begin{array}{c}
K_{v \min }\|r\|+k_{M}\|r\| W_{M}\left\|\left(\left\|W_{F}\right\|_{F}-W_{M \max }\right)+k_{V}\right\| r\|\|_{V} \|_{F}\left(\left\|W_{V}\right\|_{F}-W_{V \max }\right) \\
\quad+k_{G}\|r\| W_{G}\left\|_{F}\left(\left\|W_{G}\right\|_{F}-W_{G \max }\right)+k_{F}\right\| r\|\|_{F} \|\left(\left\|W_{F}\right\|_{F}-W_{F \max }\right)
\end{array}\right]
\end{aligned}
$$

Due to

$$
k\left\|W_{F}\right\|_{F}\left(\left\|W_{F}\right\|_{F}-W_{\max }\right)=k\left(\left\|W_{F}\right\|_{F}-W_{\max } / 2\right)^{2}-k W_{\max }^{2} / 4
$$

In order to ensure $L<0$, then

$$
\begin{aligned}
& \|r\|>\frac{k_{M} W_{\max }^{2} / 4+k_{V} W_{\max }^{2} / 4+k_{G} W_{\max }^{2} / 4+k_{F} W_{\max }^{2} / 4}{K_{v \min }} \\
& \left\|W_{M}\right\|_{F}>W_{M \max } \text { and }\left\|W_{V}\right\|_{F}>W_{V \max } \\
& \text { and }\left\|W_{G}\right\|_{F}>W_{G \max } \text { and }\left\|W_{F}\right\|_{F}>W_{F \max }
\end{aligned}
$$

\section{Numerical Simulation}

In order to verify the effectiveness of the proposed algorithm in this paper, take the robot arm system with two joints as the research object, we realized the RBF neural network adaptive control strategy based on sub-block approximation algorithm in MATLAB, the dynamics model for the robot arm system with two joints was:

$$
M(q) \ddot{q}+V(q, \dot{q}) \dot{q}+G(q)+F(\dot{q})+\tau_{d}=\tau
$$

Where

$$
\begin{aligned}
& M(q)=\left[\begin{array}{cc}
p_{1}+p_{2}+2 p_{3} \cos q_{2} & p_{2}+p_{3} \cos q_{2} \\
p_{2}+p_{3} \cos q_{2} & p_{2}
\end{array}\right], \\
& V(q, \dot{q})=\left[\begin{array}{cc}
-p_{3} \dot{q}_{2} \sin q_{2} & -p_{3}\left(\dot{q_{1}}+\dot{q}_{2}\right) \sin q_{2} \\
p_{3} q_{1} \sin q_{2} & 0
\end{array}\right] \\
& G(q)=\left[\begin{array}{c}
p_{4} g \cos q_{1}+p_{5} \cos \left(q_{1}+q_{2}\right) \\
p_{5} g \cos \left(q_{1}+q_{2}\right)
\end{array}\right], \\
& F(\dot{q})=0.02 \operatorname{sgn}(\dot{q}), \quad \tau_{d}=\left[\begin{array}{ll}
0.2 \sin (t) & 0.2 \sin (t)
\end{array}\right]^{T} .
\end{aligned}
$$


Control algorithm is realized by using Simulink and S function. Adopt the RBF neural network adaptive control strategy based on integral approximation for uncertainness, in this algorithm take (12) as the control law,(18) as adaptive law,in the RBF neural network adaptive control strategy based on sub-block approximation algorithm, take (39) as the control law,(43-46) as adaptive laws .The simulation results were shown in fgiure1 to Figure 6.Figure 1 and Figure 2 are position tracking by using the RBF neural network adaptive control strategy based on integral approximation for uncertainness and the RBF neural network adaptive control strategy based on sub-block approximation algorithm, Figure 3 and Figure 4 are input of the RBF neural network adaptive control strategy based on integral approximation for uncertainness and the RBF neural network adaptive control strategy based on sub-block approximation algorithm, Figure 5 and Figure 6 are approximation results.
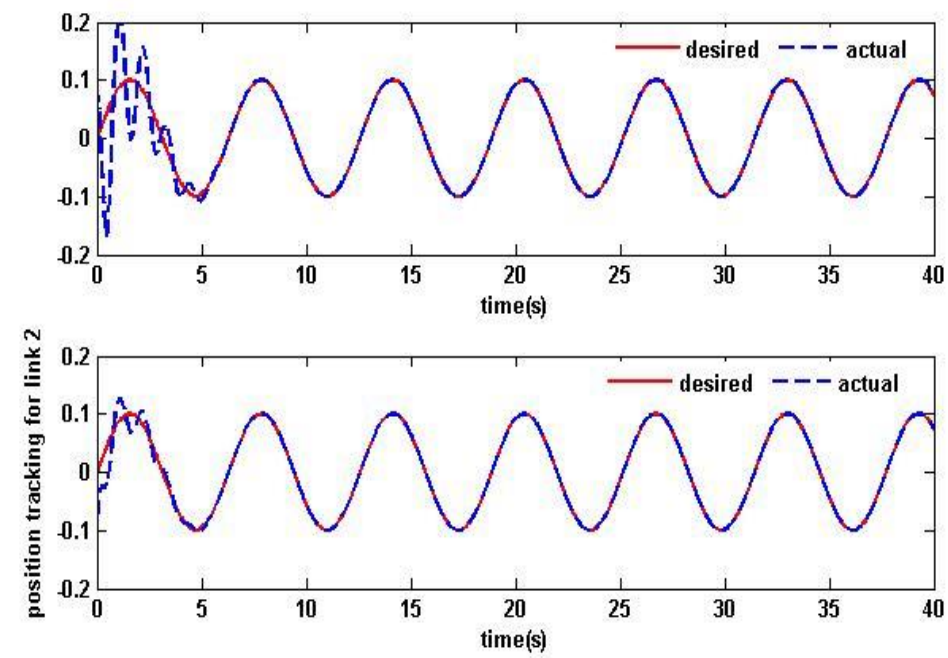

Figure 1. Position Tracking by Using the RBF Neural Network Adaptive Control Strategy Based On Integral Approximation for Uncertainness
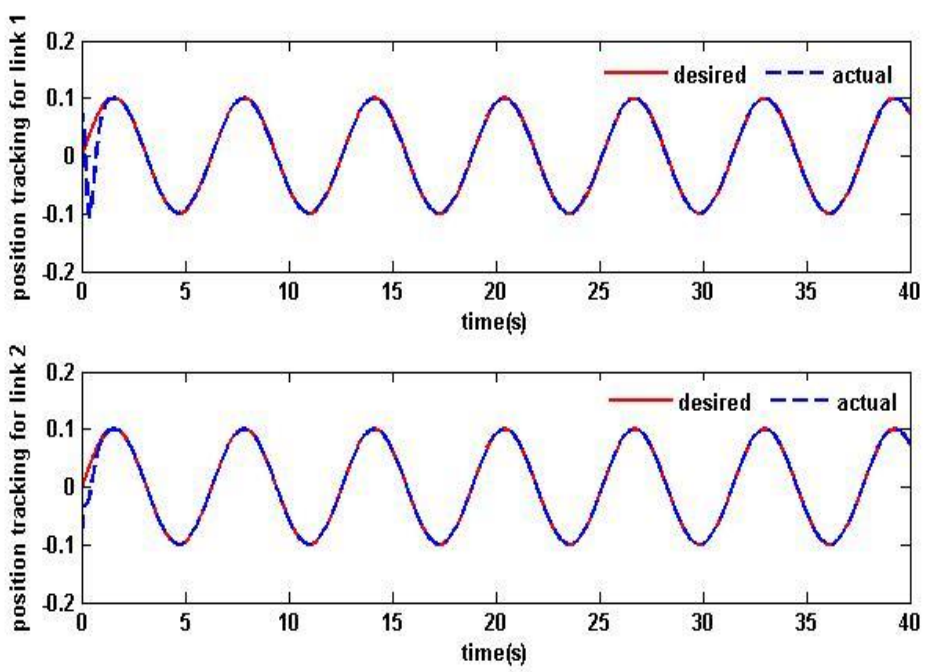

Figure 2. Position Tracking By Using the RBF Neural Network Adaptive Control Strategy Based On Sub-Block Approximation Algorithm 

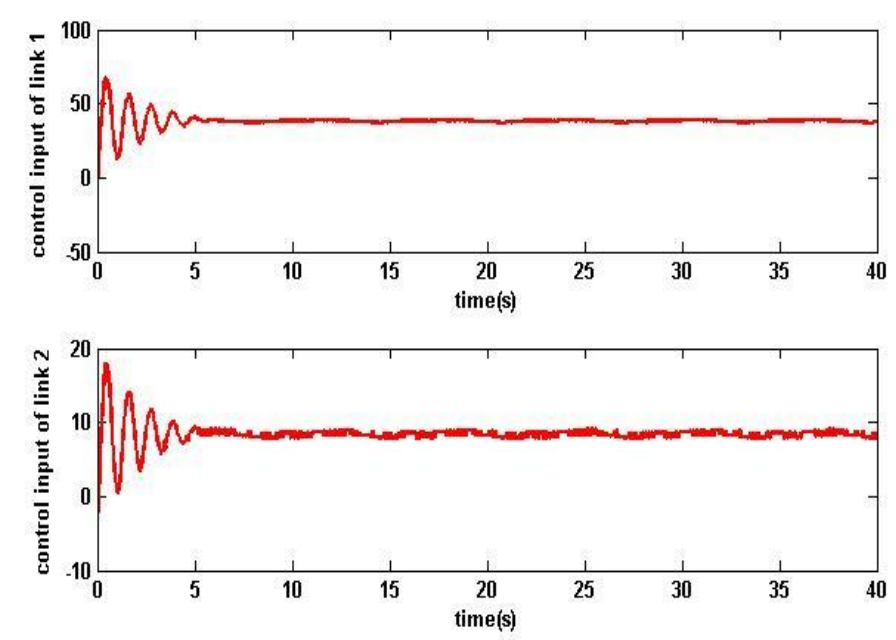

Figure 3. Input of the RBF Neural Network Adaptive Control Strategy Based On Integral Approximation for Uncertainness
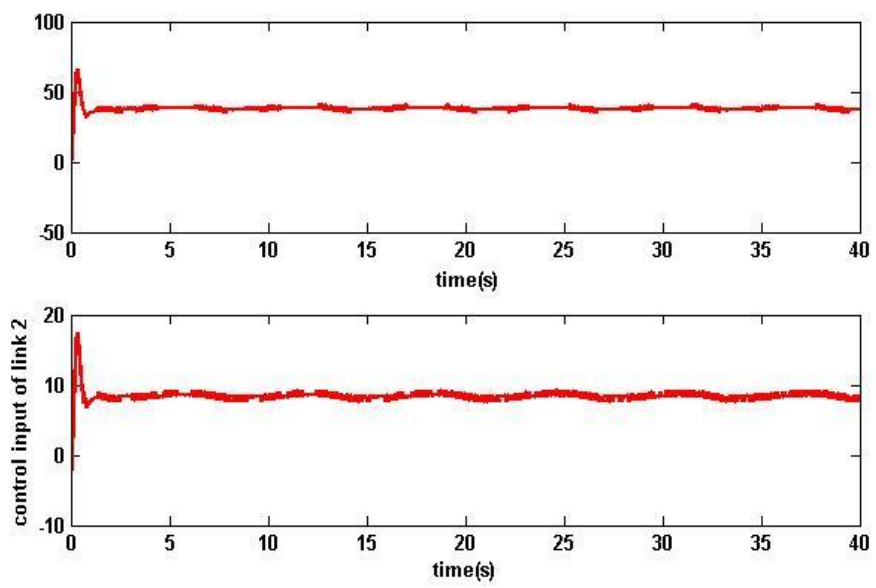

Figure 4. Input of the RBF Neural Network Adaptive Control Strategy Based On Sub-Block Approximation Algorithm

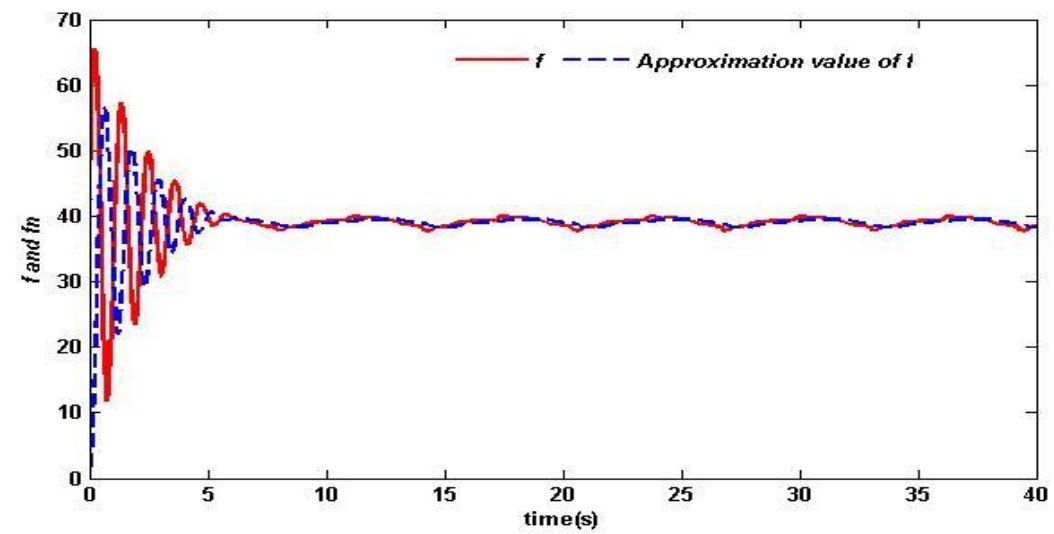

Figure 5. Approximation Results by Using the RBF Neural Network Adaptive Control Strategy Based On Sub-Block Approximation Algorithm 


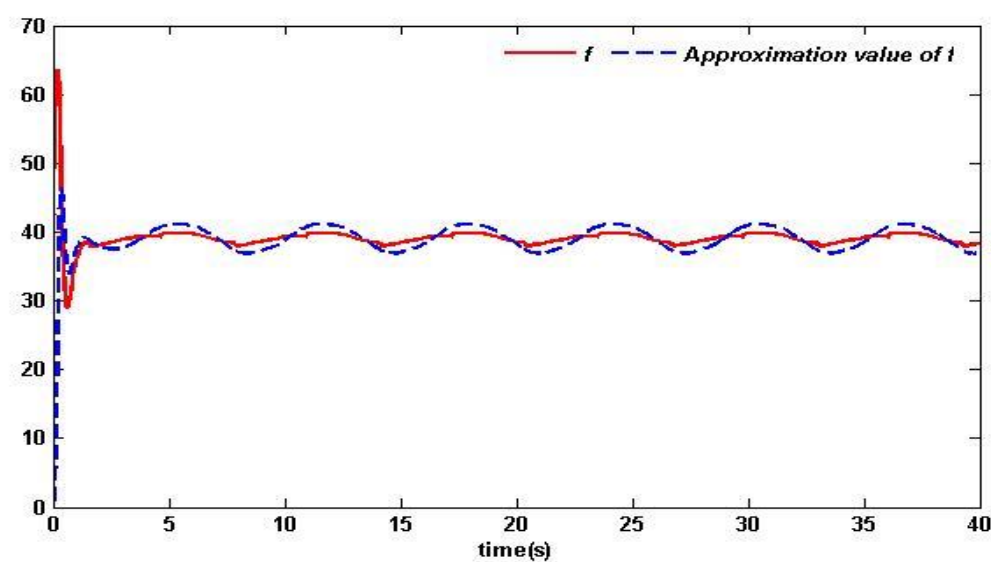

Figure 6. Approximation Results by Using the RBF Neural Network Adaptive Control Strategy Based On Integral Approximation for Uncertainness

From the Figure 1 and Figure 2 we can find that, compared with the RBF neural network adaptive control strategy based on integral approximation for uncertainness, the proposed control method has features with better convergence at $0-5 \mathrm{~S}$, there have chattering phenomenon in the RBF neural network adaptive control strategy based on integral approximation for uncertainness, Figure 3 and Figure 4 revealed the input of the $\mathrm{RBF}$ neural network adaptive control strategy based on integral approximation for uncertainness and the RBF neural network adaptive control strategy based on sub-block approximation algorithm, they shown that compared with the RBF neural network adaptive control strategy based on integral approximation for uncertainness, the proposed control method has features with smooth movement.

\section{Conclusions}

Aiming at the uncertainties that existed in the dynamic model of robot may cause instability. This paper has proposed a RBF neural network adaptive control strategy based on sub-block approximation algorithm,

(1)In this strategy, sliding model control was used to control trajectory of the joints of robot, and utilize the RBF neural network to approximate the each uncertain in the dynamic model of robot.

(2)We have proved the stability of the proposed control algorithm based on the lyapunov stability theory.

(3) In order to verify the effectiveness of the proposed algorithm in this paper, we realized the RBF neural network adaptive control strategy based on sub-block approximation algorithm in MATLAB, compared with the RBF neural network adaptive control strategy based on integral approximation for uncertainness, the proposed control method has features with better convergence at $0-5 \mathrm{~S}$, there have chattering phenomenon in the RBF neural network adaptive control strategy based on integral approximation for uncertainness, the proposed control method has features with good position tracking, the smooth movement, effective approaches, etc.

\section{Acknowledgments}

This work is supported by the Science and Technology Plan Projects of Henan Province for contract 132300410485 and 142300410463, the Key Research Projects of Universities in Henan Province for contract 15A520035 and 15A520124, under which the present work was possible. 


\section{References}

[1] H. G. Tanner, S. G. Loizou and K. J.Jkyriakopoulos, "Non-holonomic navigation and control of cooperating mobile manipulators", IEEE Transactions on Robotic Automation, vol. 19, no. 1, (2003), pp. 53-64

[2] X. Li, K. M. Yang and Y. Zhu, "Self-adaptive Control of Robotic Manipulator Based on Modeling Error Compensated by RBF", Journal of System Simulation, vol. 24, no.7, (2012), pp. 1474-1478.

[3] A. Tayeb, "Adaptive iterative learning control for robot manipuators", Automatica, vol. 40, no. 7, (2004), pp. 1159-1203.

[4] S. Liuzzoand and P. Tomei, A global adaptive learning control for robotic manipulators, Automatica, vol. 44, no. 8, (2012), pp. 1379-1384

[5] S. Liuzzo, R. Marino and P. Tomei, "Adaptive learning control of nonlinear systems by output error feedback", IEEE Transactions on Automatic Control, 007, 52 (4): 1232 - 1248, vol. 52, no. 4, (2007), pp. $1232-11248$.

[6] J. Shi, H. Liu and N. Bajcinca, Robust control of robotic manipulators based on integral sliding mode, International Journal of Control, vol. 81, no. 10, (2007), pp. 1537-1548.

[7] O. Barambones and V. Etxebarria, "Robust neural control for robotic manipulators", Automatica, vol. 38, no.2, (2002), pp. 235-242

[8] T. H. S. Li and Y. C. Huang, "MIMO adaptive fuzzy terminal sliding mode controller for robotic manipulators", Information Sciences, vol. 180, no. 23, (2010), pp. 4641-4660.

[9] Y. Zuo, Y. N. Yang and X. Z. Liu, "Neural network robust $\mathrm{H} \infty$ tracking control strategy for robot manipulators", Applied Mathematical Modelling, vol. 34, no. 7, (2010), pp. 4641-4660

[10] Z. J. Li, S. S GE and Z. P. Wang, "Robust adaptive control of coordinated multiple mobile manipulators", Mechatronics, vol. 18, no. 5, (2008), pp. 239-250.

[11] Y. N. Wang, "Robot intelligent control engineering", Science press, Beijing, China, (2004).

[12] Y. L. Liu and J. K. Sun, "Actuator Nonlinearities Compensation Using RBF Neural Networks in Robot Control System”, Computational Engineering in Systems Applications (S2223-9812), vol. 4, no. 5, (2006), pp. 231-238.

[13] J. Yu, J. LI, X. F. Wang and Y. L. Han, "Robust Adaptive Controller Design for Robot Based on RBF Neural Network", Journal of Naval Aeronautical Engineering Institute, vol. 24, no. 3, (2009), pp. 267 271

[14] L. X. Chen, "Adaptive control of robot based on RBF network with uncertainty of model approximation”, Electronic Design Engineering, vol. 201, no. 20, (2012), pp.80-83

\section{Authors}

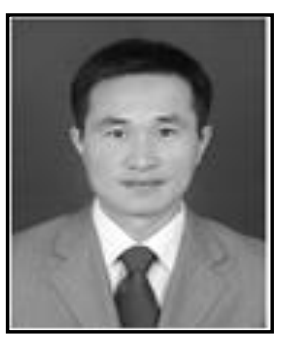

Yongfeng Cui (Corresponding author), he received the MS degree in Computer Application Technology from Huazhong University of Science and Technology, China in 2007. Now he is an associate professor at school of science and technology, Zhoukou normal university. $\mathrm{He}$ is currently researching on Computer Application Technology (CAT)

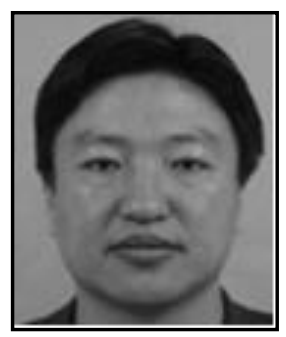

Chong Tian, he received the MS degree in Applied Mathematics from Henan Normal University, China in 2010. Now he is an associate professor at school of mathematics and statistics, Zhoukou normal university.He is currently researching on Applied Mathematics (AM). 